\title{
Synthesis of Constrained nR Planar Robots to Reach Five Task Positions
}

\author{
Gim Song Soh \\ Robotics and Automation Laboratory \\ University of California \\ Irvine, California 92697-3975 \\ Email: gsoh@uci.edu
}

\author{
J. Michael McCarthy \\ Department of Mechanical and Aerospace Engineering \\ University of California \\ Irvine, California 92697-3975 \\ Email: jmmccart@uci.edu
}

\begin{abstract}
In this paper, we design planar $\mathrm{nR}$ serial chains that provide one degree-of-freedom movement for an end-effector through five arbitrarily specified task positions. These chains are useful for deployment linkages or the fingers of a mechanical hand.

The trajectory of the end-effector pivot is controlled by n-1 sets of cables that are joined through a planetary gear system to two input variables. These two input variables are coupled by a four-bar linkage, and the movement of the end-effector around its end joint is driven by a second four-bar linkage. The result is one degree-of-freedom system.

The design of the cabling system allows control of the shape of the chain as it moves through the task positions. This combines techniques of deployable linkage design with mechanism synthesis to obtain specialized movement with a minimum number of actuators. Two example designs for a $6 R$ planar chain are presented, one with a square initial configuration and a second with a hexagonal initial configuration.
\end{abstract}

\section{INTRODUCTION}

In this paper, we present a methodology for the design of planar $\mathrm{nR}$ serial chains such that the end-effector reaches five arbitrary task positions with one degree-of-freedom. The method is general in that we may introduce mechanical constraints between $m$ drive joints and $n-m$ driven joints. For the purposes of this paper, we consider $m=1$, and show how to use cables combined with planetary gear trains to constrain the end-effector movement to three degrees-of-freedom. Then, we design four-bar chains attached at the base and at the endeffector to obtain a one degree-of-freedom system. See Figure 1. As an example, we obtain a one degree-of-freedom $6 \mathrm{R}$ planar chain that reaches five specified task positions. This work can be used to control the shape of deployable chains, as well as for the design of mechanical fingers for specialized grasping.

\section{Literature REview}

Cable systems are employed to reduce the inertia of a manipulator system by permitting the actuators to be located remotely, thus a higher power-to-weight ratio over a direct drive system, see Lee (1991) [1]. Recent work on cabledriven robotic systems includes Arsenault and Gosselin (2006) [2], who introduce a 2-dof tensegrity mechanism to increase the reachable workspace and task flexibility of a serial chain robot. Also, Lelieveld and Maeno (2006) [3] present a 4 DOF

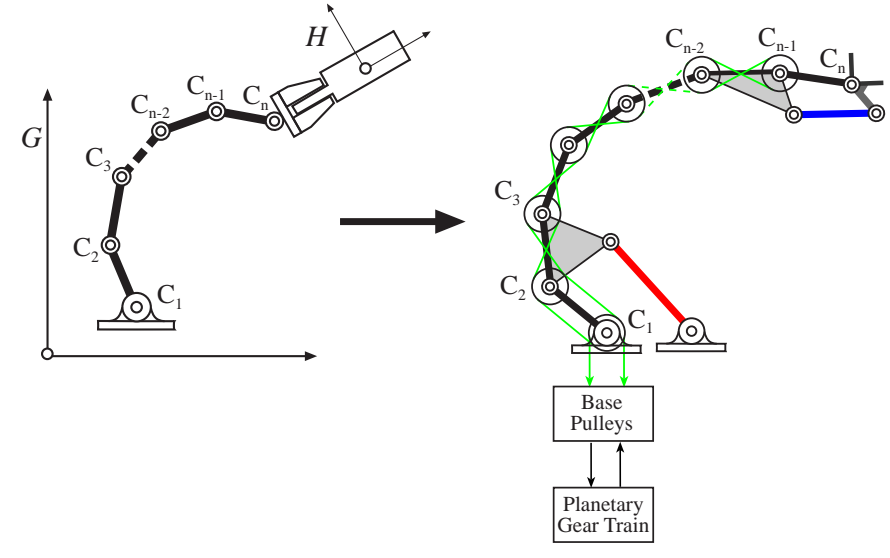

Fig. 1. The Design Problem. The figure on the left shows a schematic of planar $\mathrm{nR}$ serial robot, and the figure on the right shows a schematic of the constrained $\mathrm{nR}$ robot.

portable haptic device for the index finger driven by a cableactuated rolling-link-mechanism (RLM). Palli and Melchiorri (2006) [4] explore a variation on cables using a tendon sheath system to drive a robotic hand.

Our work is inspired by Krovi et al. (2002) [5], who present a synthesis theory for $n \mathrm{R}$ planar serial chains driven by cable tendons. Their design methodology sizes the individual links and pulley ratios to obtain the desired end-effector movement used to obtain an innovative assistive device. Our approach borrows from deployable structures research Kwan and Pelligrino (1994) [6], and uses symmetry conditions to control the overall movement of the chain, while specialized RR cranks position the base and end-effector joints.

For our serial chain the designer can choose the location of the base joint $\mathbf{C}_{1}$, the attachment to the end-effector $\mathbf{C}_{n}$, and the dimensions of the individual links which for convenience we assume are the same size. See Figure 1. Our goal is to design the mechanical constraints that ensures the end-effector reaches each of five task positions, in response to an input drive variable. This research extends the ideas presented in Leaver and McCarthy (1987) [7] and Leaver et al. (1988) [8] using the tendon routing analysis of Lee (1991) [1]. Also see Tsai (1999) [9]. While our proposed system appears complicated, 
it has only one actuator.

\section{The Planar nR Serial Chain Robot}

Let the configuration of an $\mathrm{nR}$ serial chain be defined by the coordinates $\mathbf{C}_{i}=\left(x_{i}, y_{i}\right), i=1, \ldots, n$ to be each of its revolute joints. See Figure 1. The distances $a_{i, i+1}=\mid \mathbf{C}_{i+1}-$ $\mathbf{C}_{i} \mid$ defined the lengths of each link. Attach a frame $B_{i}$ to each of these links so its origin is located at $\mathbf{C}_{i}$ and its $x$ axis is directed toward $\mathbf{C}_{i+1}$. The joints $\mathbf{C}_{1}$ and $\mathbf{C}_{n}$ are the attachments to the base frame $F=B_{1}$ and the moving frame $M=B_{n}$, respectively, and we assume they are the origins of these frames. The joint angles $\theta_{i}$ define the relative rotation about the joints $\mathbf{C}_{i}$.

Introduce a world frame $G$ and task frame $H$ so the kinematics equations of the $\mathrm{nR}$ chain are given by

$$
[D]=[G]\left[Z\left(\theta_{1}\right)\right]\left[X\left(a_{12}\right)\right] \ldots\left[X\left(a_{n-1, n}\right)\left[Z\left(\theta_{n}\right)\right][H],\right.
$$

where $\left[Z\left(\theta_{i}\right)\right]$ and $\left[X\left(a_{i, i+1}\right)\right]$ are the $3 \times 3$ homogeneous transforms that represent a rotation about the z-axis by $\theta_{i}$, and a translation along the x-axis by $a_{i, i+1}$, repspectively. The transformation $[G]$ defines the position of the base of the chain relative to the world frame, and $[H]$ locates the task frame relative to the end-effector frame. The matrix $[D]$ defines the coordinate transformation from the world frame $G$ to the task frame $H$.

\section{Kinematics of TENDON DRIVEn Robots}

The relationship between the rotation of the base pulley and the joint angles in an open-loop chain with $(k+1)$ links can be described by:

$$
\theta_{k}^{*}=\theta_{1} \pm\left(\frac{r_{k, 2}}{r_{k}}\right) \theta_{2} \pm \ldots\left(\frac{r_{k, i}}{r_{k}}\right) \theta_{i} \pm \ldots\left(\frac{r_{k, k}}{r_{k}}\right) \theta_{k}
$$

where $\theta_{k}^{*}$ denotes the base pulley angle of the $k^{t h}$ tendon, $\theta_{i}$ denotes the joint angle of the $i^{t h}$ joint, $r_{k, i}$ denotes the radius of the pulley of the $i^{t h}$ joint of the $k^{t h}$ tendon, and $r_{k}$ denotes the radius of the base pulley of the $k^{\text {th }}$ tendon. The sign of each term, $\left(\frac{r_{k, i}}{r_{k}}\right) \theta_{i}$, in (2) is to be determined by the number of cross-type routing preceding the $i^{t h}$ joint axis. If the number of cross-type routing is even, the sign is positive, else it is negative. See the connection between $\mathbf{C}_{1}$ and $\mathbf{C}_{2}$ of Tendon 3 in Figure 2 for an example of cross-type routing. For derivation of this equation, refer to Lee (1991) [1].

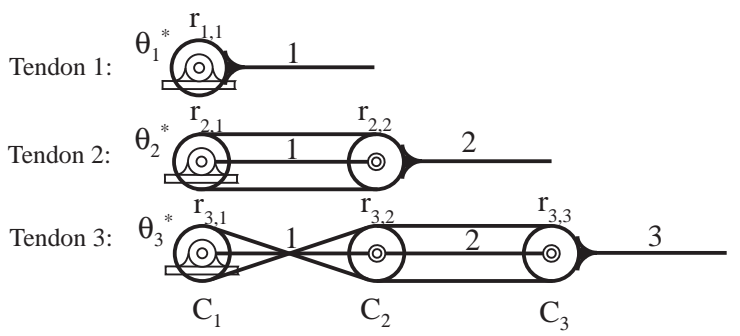

Fig. 2. Planar schematic for a three-DOF planar robot with structure matrix (5).
Writing (2) once for each of the tendon routing $k, k=$ $1, \ldots, n$ for an $\mathrm{nR}$ serial robot, yields the following linear transformation in matrix form:

$$
\boldsymbol{\Theta}^{*}=[B][R] \Theta .
$$

where $\boldsymbol{\Theta}^{*}=\left(\theta_{1}^{*}, \theta_{2}^{*}, \ldots, \theta_{n}^{*}\right)^{T}$ denotes the base pulley angles and $\boldsymbol{\Theta}=\left(\theta_{1}, \theta_{2}, \ldots, \theta_{n}\right)^{T}$ denotes the joint angles. If we let $r_{k, j}=r_{k}, k=1, \ldots, n$ such that all the pulleys have the same diameter, then $[B][R]$ would be reduced to a lower triangular $n \times n$ matrix with entries +1 or -1 ,

$$
[B][R]=[B]=\left[\begin{array}{ccccc}
1 & 0 & 0 & \ldots & 0 \\
1 & \pm 1 & 0 & \ldots & 0 \\
\vdots & \vdots & \vdots & \ldots & \vdots \\
1 & \pm 1 & \pm 1 & \ldots & \pm 1
\end{array}\right]
$$

The matrix $[B]$ is known as the structure matrix, Lee (1991) [1]. It describes how the tendons are routed. The matrix $[R]$ is a diagonal scaling matrix with elements representing the ratio of the radii of the $i$-th joint pulley to the $i$-th base pulley. In this paper, we size the pulleys to be of equal lengths such that this matrix is the identity.

We illustrate with an example on constructing the tendons of a $3 \mathrm{R}$ serial robot from the structure matrix. Suppose the structure matrix, $[B]$ is defined to be

$$
[B]=\left[\begin{array}{ccc}
1 & 0 & 0 \\
1 & 1 & 0 \\
1 & -1 & -1
\end{array}\right] .
$$

Each columns of the matrix $[B]$ corresponds to the routing prior to the joint pivots, and each row represent an independent tendon drive. The element in the first row is always +1 since we are driving the fixed pivot $\mathbf{C}_{1}$ directly with $\theta_{1}^{*}$. For the second row, it represent a parallel-type routing between the moving pivot $\mathbf{C}_{2}$ and fix pivot $\mathbf{C}_{1}$ since the sign of the second element remains unchanged. For the last row, the second element changes sign, we get a cross-type between the moving pivot $\mathbf{C}_{2}$ and fix pivot $\mathbf{C}_{1}$. Also, the sign remains unchanged after the second element, parallel-type follows between the moving pivot $\mathbf{C}_{3}$ and $\mathbf{C}_{2}$. See Figure 2 for the planar schematic for the structure matrix (5).

\section{Schematic of A CONSTRAin nR Planar Robot}

The schematic of a constrain nR Planar Robot consists of a nR serial chain, $N-1$ tendons, a planetary gear train, and two RR chains. See Figure 3. Our goal is to use the $N-1$ tendons, a planetary gear train, and two RR chains to mechanically constrain a specified $\mathrm{nR}$ serial chain that reaches five task position to one degree-of-freedom. The $N-1$ base pulleys were connected to the $N-1$ tendons to group the actuation point to the base for easy attachment to a planetary gear train.

The specified $\mathrm{nR}$ serial chain have $\mathrm{n}$ degrees of freedom. The attachment of the $N-1$ tendons to the $\mathrm{nR}$ serial chain does not change the system degrees of freedom, but it allows the control of the first $n-1$ joint angles from the base independently. If we add a planetary gear train to couple the various $n-1$ base pulleys as shown in Figure 5, we are able 


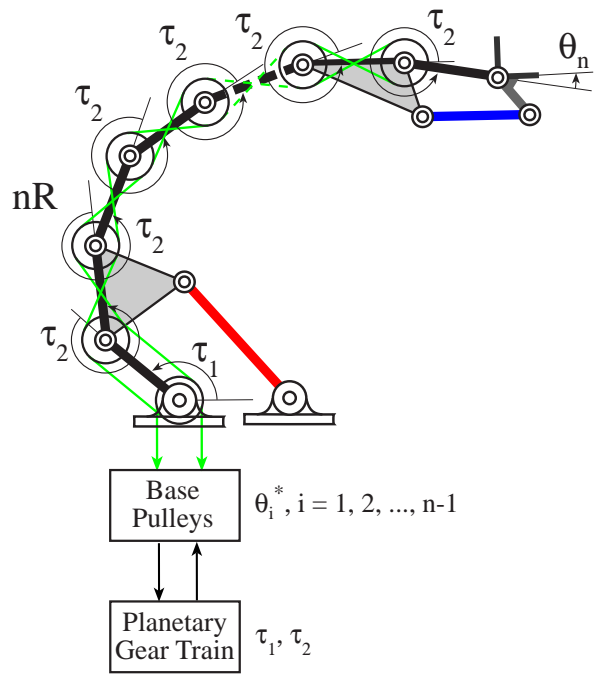

Fig. 3. Schematic for a constrain $\mathrm{nR}$ Planar Robot driven by cables, mechanically constraint by planetary gear train and RR cranks.

to reduce the tendon driven robot to a three degree-of-freedom system. Two RR constraints were then added to obtain a one degree-of-freedom system. The first RR chain serves to couple the planetary gear train inputs $\tau_{1}$ and $\tau_{2}$ together, and the purpose of the second RR chain is to couple the $n$th joint, $\theta_{n}$, to $\tau_{2}$ and hence $\tau_{1}$.

As shown in Figure 3, if we denote $\tau_{1}$ as the input that drive the first joint angle, we can see that $\tau_{2}$ is related to the input $\tau_{1}$ through the four bar linkage formed from the attachment of an RR constraint to the second link. The planetary gear train was designed in such a way that it impose symmetry condition on the joint angles so that $\theta_{2}=\theta_{3}=\cdots=\theta_{n-1}=\tau_{2}$. Hence $\tau_{2}$ get reflected through the tendons to serve as the input to the floating four bar linkage from the RR attachment between the end-effector and the $(n-2)$ th link. The output from this floating four-bar linkage is the joint angle $\theta_{n}$. In other words, if we drive the system with input $\tau_{1}, \tau_{2}$ and $\theta_{n}$ will be driven by the input $\tau_{1}$ through the mechanical coupling of the planetary gear train, and the two RR constraints.

\section{The Coupling Between Joint Angles And Inputs}

The desired coupling between joint angles, $\Theta$, and the inputs, $\Gamma$, of a $\mathrm{nR}$ serial chain can be described by matrix $[A]$ as follows,

$$
\Theta=[A] \Gamma
$$

Our goal here is to constrain the $\mathrm{nR}$ serial chain to a three degree-of-freedom system first using tendons and a planetary gear train before we add RR chainss to constrain it to one degree-of-freedom. Hence $\Gamma=\left(\tau_{1}, \tau_{2}\right)^{T}$ in particular if we ignore $\theta_{n}$ for now. The desired coupling between the inputs and the joint angles for a $\mathrm{nR}$ serial planar robot can be obtained by specifying the tendon routing, the size of the base pulleys, and the transmission coupling the input to the base pulleys. This is equivalent to selecting the matrices $[B],[S]$, and $[C]$ so we obtain the desired coupling matrix $[\mathrm{A}]$ in the relation

$$
[A]=[B]^{-1}[S][C] .
$$

$[B]$ is a matrix that describes the relationship between the relative joint angles and the rotation of the base pulleys, see (3). $[S]$ is a diagonal matrix that scales the sum of the row elements of matrix $[C]$ to unity. This matrix was included to account for the effects of changing the size of the base pulleys and is the ratio of the $i^{t h}$ base pulley to the $i^{\text {th }}$ joint pulley. $[C]$ is a matrix that describes the relationship between the rotation of the base pulleys to the inputs of the transmission system.

\section{THE TRANSMISSION SySTEM}

The transmission that couples the $\mathrm{nR}$ serial robot is composed of two stages. The bottom stage connects the controlled inputs to the base pulleys using simple and planetary gear trains and the top stage connects the base pulleys to the joint pulleys using tendons.

The relationship between the inputs, $\Gamma$ and the base pulley rotations $\Theta^{*}$, which defines the bottom stage of the transmission is given by:

$$
\Theta^{*}=[C] \Gamma .
$$

The matrix $[C]$ is called the mechanical coupling matrix. Its elements determine the gear train design which couples the inputs to the tendon drive pulleys. In this paper, our goal is to first design a transmission system that couples the $n R$ serial chain to three degree-of-freedom, and then design two RR cranks to constraint the system to one degree-of-freedom. The equation relating the base pulley rotation, $\theta_{i}^{*}$ to the inputs at the transmission $\tau_{1}$ and $\tau_{2}$ is:

$$
\theta_{i}^{*}=c_{i} \tau_{1}+d_{i} \tau_{2}
$$

Three cases exist for (9). They correspond to a simple gear train if either $c_{i}$ or $d_{i}=0$, a planetary gear train if both $c_{i}$ and $d_{i}$ are non-zero, and un-driveable if both $c_{i}$ and $d_{i}$ are zero. An example of a planetary gear train with sum of $c_{i}=1$ and $d_{i}=3$ is as shown in Figure 4.

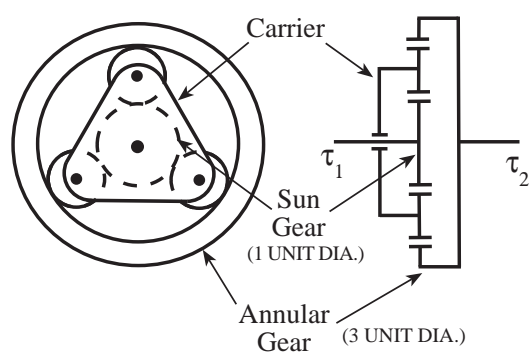

Fig. 4. Planetary Gear Train

\section{NR ChAIN DESIGN Methodology}

Our design of constraining $\mathrm{nR}$ robots using tendon, planetary gear train and RR chainss to reach five specified task positions proceed in three steps. The following describes the design process. 
A. Step 1 - Solving the Inverse Kinematics of the $n R$ Serial Chain

Given five task positions $\left[T_{j}\right], j=1, \ldots, 5$ of the endeffector of the $\mathrm{nR}$ chain, we seek to solve the equations

$$
[D]=\left[T_{j}\right], \quad j=1, \ldots, 5,
$$

to determine the joint parameter vectors $\boldsymbol{\Theta}_{j}=$ $\left(\theta_{1, j}, \theta_{2, j}, \ldots, \theta_{n, j}\right)^{T}$, where $\theta_{i, j}$ represents the $i^{t h}$ joint angle at the $j^{\text {th }}$ position. Because there are three independent constraints in this equation, we have free parameters when $n>3$. In what follows, we show how to use these free parameters to facilitate the design of mechanical constraints on the joint movement so the end-effector moves smoothly through the five task positions.

In the design of serial chain robots, it is convenient to have near equal length links to reduce the size of workspace holes. For this reason, we assume that the link dimensions of our $\mathrm{nR}$ planar robot satisfy the relationship

$$
a_{12}=a_{23}=\cdots=a_{n-1, n}=l .
$$

This reduces the specification of the $\mathrm{nR}$ robot to the location of the base joint $\mathbf{C}_{1}$ in $G$, and the end-effector joint $\mathbf{C}_{n}$ relative to the task frame $H$. In this paper, we take the task frame $H$ to be the identity matrix to simplify the problem even more. The designer is free to chose the location of the base joint $\mathbf{C}_{1}$ and the link dimensions $l$.

We define the input crank joint angle to be,

$$
\theta_{1, j}=\sigma_{j}, \quad j=1, \ldots, 5,
$$

and if the planar $\mathrm{nR}$ robot has $n>3$ joints, then we impose a symmetry condition

$$
\theta_{2, j}=\theta_{3, j}=\cdots=\theta_{n-1, j}=\lambda_{j}, \quad j=1, \ldots, 5,
$$

in order to obtain a unique solution to the inverse kinematics equations.

\section{B. Step 2 - Designing of a planetary gear train}

The second step of our design methodology consists of designing a two-degree-of-freedom planetary gear train to drive the base pulleys to achieve the symmetry conditions listed in (13). Note that we ignore the degree of freedom of the $n^{t h}$ joint at this stage. The gear train controls the shape of the chain through the two inputs. Proper selection of the routing of the tendons from their joints to their base pulley can simplify gear train design. We now generalize the routing of the $n-1$ tendon such that we only require a planetary gear train with inputs $\tau_{1}$ and $\tau_{2}$ to drive the first $n-1$ joint angles. Consider the following $(n-1) \times 2$ desired coupling matrix

$$
[A]=\left[\begin{array}{cc}
1 & 0 \\
0 & 1 \\
\vdots & \vdots \\
0 & 1
\end{array}\right]
$$

If we chose the $(n-1) \times(n-1)$ structure matrix $[B]$ with elements such that starting from the second column, we have
+1 on the even columns and -1 on the odd columns to get the following lower triangular matrix,

$$
[B]=\left[\begin{array}{ccccccc}
1 & 0 & 0 & 0 & 0 & \ldots & 0 \\
1 & 1 & 0 & 0 & 0 & \ldots & 0 \\
1 & 1 & -1 & 0 & 0 & \ldots & 0 \\
1 & 1 & -1 & 1 & 0 & \ldots & 0 \\
1 & 1 & -1 & 1 & -1 & \ldots & 0 \\
\vdots & \vdots & \vdots & \vdots & \vdots & \ldots & \vdots \\
1 & 1 & -1 & 1 & -1 & \ldots & \pm 1
\end{array}\right] .
$$

Refer to Figure 7 and 8 for a graph and schematic representation of such a routing system. Using (7), we get

$$
[B][A]=[S][C]=\left[\begin{array}{cc}
1 & 0 \\
1 & 1 \\
1 & 0 \\
1 & 1 \\
1 & 0 \\
\vdots & \vdots \\
c_{n-1,1} & c_{n-1,2}
\end{array}\right] .
$$

If we introduce a $(n-1) \times(n-1)$ diagonal scaling matrix,

$$
[S]=\left[\begin{array}{cccccc}
1 & 0 & 0 & 0 & \ldots & 0 \\
0 & 2 & 0 & 0 & \ldots & 0 \\
0 & 0 & 1 & 0 & \ldots & 0 \\
0 & 0 & 0 & 2 & \ldots & 0 \\
\vdots & \vdots & \vdots & \vdots & \ldots & \vdots \\
0 & 0 & 0 & 0 & \ldots & s_{n-1}
\end{array}\right],
$$

with element $s_{n-1}=1$ if $\mathrm{n}$ is even, and $s_{n-1}=2$ if $\mathrm{n}$ is odd. It would result in a mechanical coupling matrix $[C]$, with row elements adding to unity, of the form

$$
[C]=\left[\begin{array}{cc}
1 & 0 \\
\frac{1}{2} & \frac{1}{2} \\
1 & 0 \\
\frac{1}{2} & \frac{1}{2} \\
1 & 0 \\
\vdots & \vdots \\
c_{n-1,1} & c_{n-1,2}
\end{array}\right]
$$

Note that the rows of matrix $[C]$ consists of elements $(1,0)$ at odd rows and $\left(\frac{1}{2}, \frac{1}{2}\right)$ at even rows. The values at the last row would depend on if $n-1$ is odd or even. Because of this special alternating structure, we only require a two degree-of-freedom planetary gear train to drive the $\mathrm{n}-1$ tendon base pulleys.

The mechanical coupling matrix $[C]$ indicates that the planetary gear train used should have the property such that $\tau_{1}$ drives odd base joints, and $\tau_{1}$ and $\tau_{2}$ drives even base joints. A planetary gear train that fits this characteristic would be a bevel gear differential since an ordinary planetary gear train would result in a locked state. Also, the scaling matrix indicates that the ratio of the even $k^{t h}$ base pulley to the even $k^{t h}$ joint pulley is 1 to 2 . See Figure 5 and Figure 6 for an example of such a bevel gear differential and tendon routing on a $6 \mathrm{R}$ planar robot. 


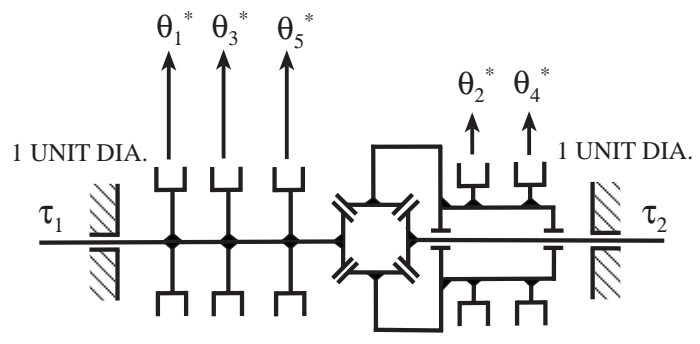

Fig. 5. Planetary Gear Train for a $6 \mathrm{R}$ Planar serial Robot

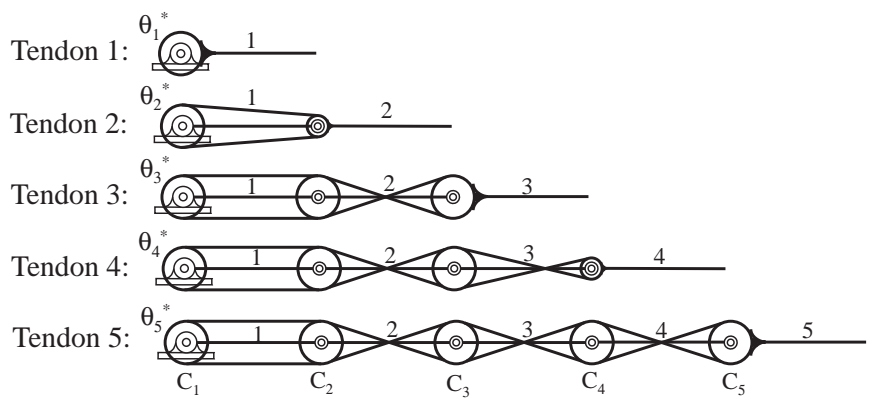

Fig. 6. Tendon Routing for a 6R Planar Robot

\section{Step 3 - Synthesis of an RR Drive Crank}

The last step of our design methodology consists of sizing two RR chainss that constrains the $\mathrm{nR}$ robot to one degree-offreedom. Figure 7 and 8 shows how we can systematically attach two RR chainss to constraint the three degree-offreedom $\mathrm{nR}$ cable driven robot to one degree-of-freedom. In this section, we expand the RR synthesis equations, Sandor and Erdman (1984) [10] and McCarthy (2000) [11], to apply to this situation.

Let $\left[B_{k-2, j}\right]$ be five position of the $(k-2)$ th moving link, and $\left[B_{k, j}\right]$ be the five positions of the $k$ th moving link measured in a world frame $F, j=1, \ldots, 5$. Let $\mathrm{g}$ be the coordinates of the R-joint attached to the $(k-2)$ th link measured in the link frame $B_{k-2}$, see Figure 9. Similarly, let $\mathbf{w}$ be the coordinates of the other R-joint measured in the link frame $B_{k}$. The five positions of these points as the two moving bodies move between the task configurations are given by

$$
\mathbf{G}^{j}=\left[B_{k-2, j}\right] \mathbf{g} \text { and } \quad \mathbf{W}^{j}=\left[B_{k, j}\right] \mathbf{w}
$$

Now, introduce the relative displacements $\left[R_{1 j}\right]=$ $\left[B_{k-2, j}\right]\left[B_{k-2,1}\right]^{-1}$ and $\left[S_{1 j}\right]=\left[B_{k, j}\right]\left[B_{k, 1}\right]^{-1}$, so these equations become

$$
\mathbf{G}^{j}=\left[R_{1 j}\right] \mathbf{G}^{1} \quad \text { and } \quad \mathbf{W}^{j}=\left[S_{1 j}\right] \mathbf{W}^{1}
$$

where $\left[R_{11}\right]=\left[S_{11}\right]=[I]$ are the identity transformations.

The point $\mathbf{G}^{j}$ and $\mathbf{W}^{j}$ define the ends of a rigid link of length $R$, therefore we have the constraint equations

$$
\left(\left[S_{1 j}\right] \mathbf{W}^{1}-\left[R_{1 j}\right] \mathbf{G}^{1}\right) \cdot\left(\left[S_{1 j}\right] \mathbf{W}^{1}-\left[R_{1 j}\right] \mathbf{G}^{1}\right)=R^{2}
$$

$3 \mathrm{R}$

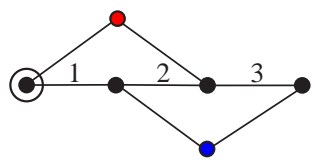

$4 \mathrm{R}$

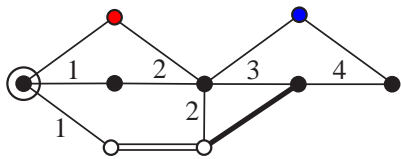

$5 \mathrm{R}$

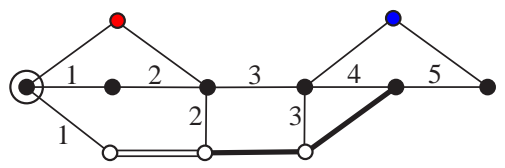

$6 \mathrm{R}$

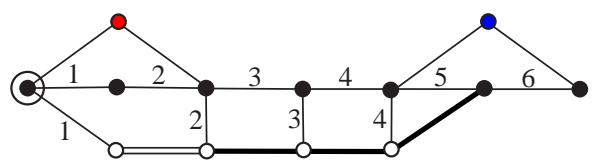

$\mathrm{nR}$

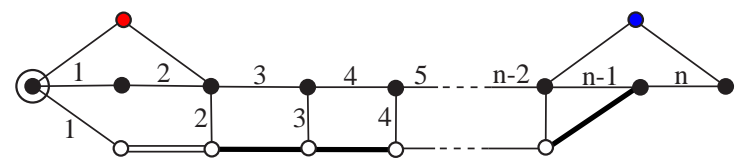

Fig. 7. This shows the graph representation of mechanically constrained serial chain. Each link is represented as a node and each $\mathrm{R}$ joint as an edge. Parallel type routing is denoted by a double-line edge and cross type routing is denoted by a heavy edge.

These five equations can be solved to determine the five design parameters of the RR constraint, $\mathbf{G}^{1}=(u, v, 1)$, $\mathbf{W}^{1}=(x, y, 1)$ and $R$. We will refer to these equations as the synthesis equations for the RR link.

To solve the synthesis equations, it is convenient to introduce the displacements $\left[D_{1 j}\right]=$ $\left[R_{1 j}\right]^{-1}\left[S_{1 j}\right]=\left[B_{k-2,1}\right]\left[B_{k-2, j}\right]^{-1}\left[B_{k, j}\right]\left[B_{k, 1}\right]^{-1}$, so these equations become

$$
\left(\left[D_{1 j}\right] \mathbf{W}^{1}-\mathbf{G}^{1}\right) \cdot\left(\left[D_{1 j}\right] \mathbf{W}^{1}-\mathbf{G}^{1}\right)=R^{2}
$$

which is the usual form of the synthesis equations for the RR crank in a planar four-bar linkage, see McCarthy (2000)[11]. Subtract the first of these equations from the remaining to cancel $R^{2}$ and the square terms in the variables $u, v$ and $x, y$. The resulting four bilinear equations can be solved algebraically, or numerically using something equivalent to Mathematica's Nsolve function to obtain the desired pivots.

The RR chain imposes a constraint on the value of $\tau_{2}$ as a function of $\tau_{1}$ given by

$$
\tau_{2}=\arctan \left(\frac{b \sin \psi-a \sin \tau_{1}}{g+b \cos \psi-a \cos \tau_{1}}\right)+\beta,
$$

where $\beta$ is the joint angle between pivots $\mathbf{C}_{2} \mathbf{W}_{1}$ and $\mathbf{C}_{2} \mathbf{C}_{3}$, 

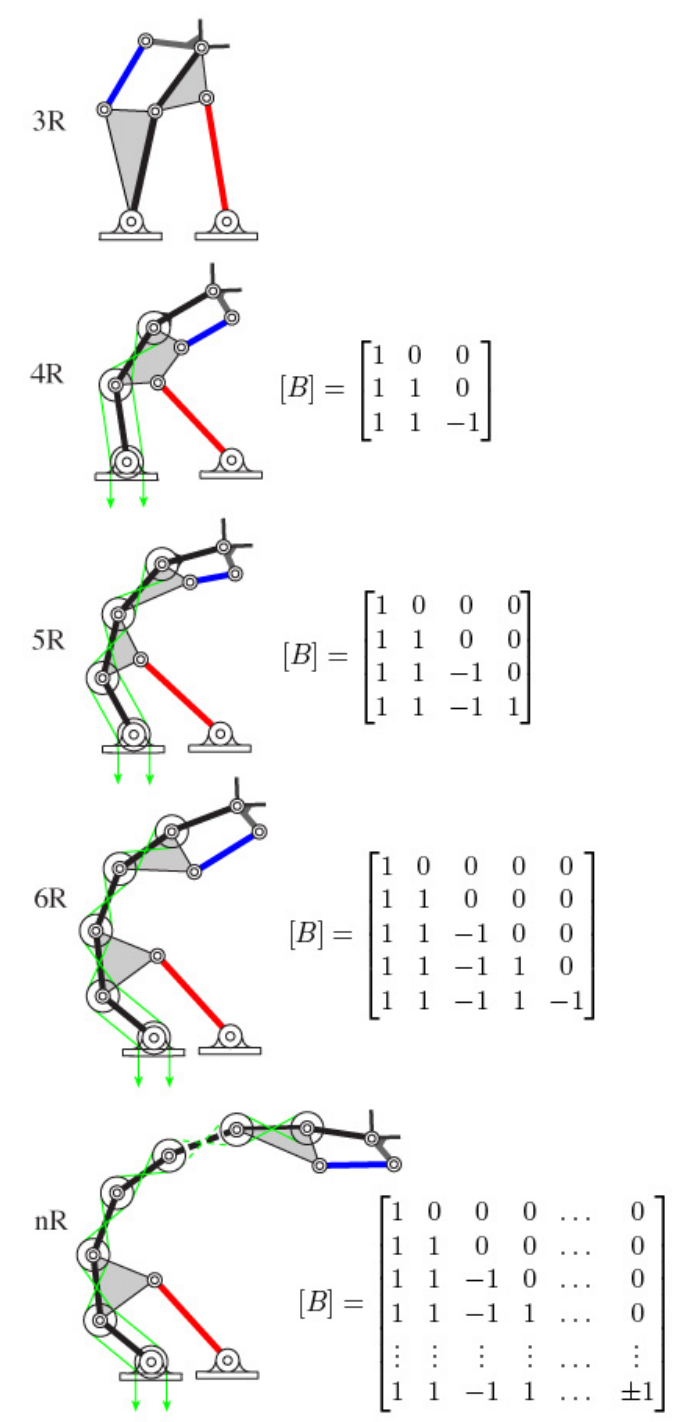

Fig. 8. This shows the kinematic structure of mechanically constrained serial chain. The matrix on the right is the structure matrix $[B]$, which describes how the tendons are routed. This show that the structure extends to any length of $\mathrm{nR}$ robot

and

$$
\begin{aligned}
\psi & =\arctan \left(\frac{B}{A}\right) \pm \arccos \left(\frac{C}{\sqrt{A^{2}+B^{2}}}\right) \\
A & =2 a b \cos \tau_{1}-2 g b \\
B & =2 a b \sin \tau_{1} \\
C & =g^{2}+b^{2}+a^{2}-h^{2}-2 a g \cos \tau_{1} .
\end{aligned}
$$

$a, b, g$ and $h$ are the lengths of link $\mathbf{C}_{1} \mathbf{C}_{2}, \mathbf{G}_{1} \mathbf{W}_{1}, \mathbf{C}_{1} \mathbf{G}_{1}$ and $\mathbf{C}_{2} \mathbf{W}_{1}$ respectively, and $\psi$ is the angle of the driven crank $b$. See Figure 9 for the various notation.

\section{CONFIGURATION ANALYSIS}

In order to evaluate and animate the movement of these linkage systems, we must analyze the system to determine its configuration for each value of the input angle $\theta_{1}$. Figure 9 shows that these systems consist of two interconnected four

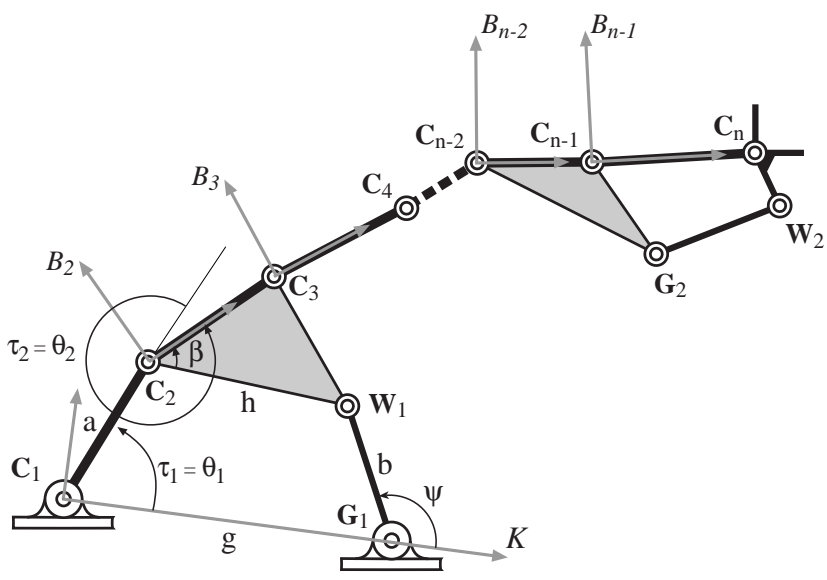

Fig. 9. This shows our conventions for the analysis of a mechanically constrained $\mathrm{nR}$ planar robot

TABLE I

FIVE TASK POSITIONS FOR THE END-EFFECTOR OF THE PLANAR 6R ROBOT WITH A SQUARE INITIAL CONFIGURATION

\begin{tabular}{|c||c|}
\hline Task & Position $(\phi, x, y)$ \\
\hline 1 & $\left(0^{\circ}, 0,-1\right)$ \\
\hline 2 & $\left(-39^{\circ}, 0.25,2\right)$ \\
\hline 3 & $\left(-61^{\circ}, 0.8,2.35\right)$ \\
\hline 4 & $\left(-94^{\circ}, 1.75,2.3\right)$ \\
\hline 5 & $\left(-117^{\circ}, 2.25,2.1\right)$ \\
\hline
\end{tabular}

bar linkages. Our approach uses the analysis procedure of four bar linkages from McCarthy (2000) [11]. Starting from frame $K$, we solve for $\mathbf{W}_{1}$ for a given $\theta_{1}$. Then, we calculate the coupler angle and hence the joint angle $\theta_{2}$ using (23). This joint angle value gets transmitted to the rest of the $n-2$ joints to drive the link $\mathbf{C}_{n-1} \mathbf{G}_{n}$. Next, we analyze the second four bar linkage in frame $B_{n-1}$ to complete the analysis. The result is a complete analysis of the mechanically constrained $\mathrm{nR}$ planar robot.

\section{DESIGN OF A 6R PLANAR CHAIN}

We illustrate how the design methodology can be used to control the shape of the deployable chain with two examples on a $6 \mathrm{R}$ planar robot, one with a square initial configuration and a second with a hexagonal initial configuration.

\section{A. Example with a square initial configuration}

Let the task positions of the planar robot in Table I be represented by $\left[T_{j}\right], j=1 \ldots, 5$. These positions represent the movement of the manipulator of the $6 \mathrm{R}$ planar robot from a square folded state to its extended state. The forward kinematics of the $6 \mathrm{R}$ serial chain is given by

$$
[D]=[G]\left[Z\left(\theta_{1}\right)\right]\left[X\left(a_{12}\right)\right] \ldots\left[X\left(a_{56}\right)\left[Z\left(\theta_{6}\right)\right][H] .\right.
$$

To solve for the inverse kinematics of the $6 \mathrm{R}$ chain, we select $\mathbf{C}_{1}=(0,0)$ such that $[G]$ becomes the identity $[I]$, and the link dimensions of the $6 \mathrm{R}$ chain $a_{12}=a_{23}=a_{34}=a_{45}=$ $a_{56}=1 \mathrm{~m}$. In addition, we assume $[H]$ to be the identity $[I]$, 
TABLE II

INVERSE KINEMATICS OF THE 6R CHAIN AT EACH TASK POSITIONS WITH A SQUARE INITIAL CONFIGURATION

\begin{tabular}{|c||c||c||c|}
\hline Task & $\sigma_{j}$ & $\lambda_{j}$ & $\theta_{6, j}$ \\
\hline 1 & $270^{\circ}$ & $-90^{\circ}$ & $90^{\circ}$ \\
\hline 2 & $181.26^{\circ}$ & $-49.19^{\circ}$ & $-23.49^{\circ}$ \\
\hline 3 & $159.75^{\circ}$ & $-44.28^{\circ}$ & $-43.65^{\circ}$ \\
\hline 4 & $132.50^{\circ}$ & $-39.88^{\circ}$ & $-66.97^{\circ}$ \\
\hline 5 & $118.62^{\circ}$ & $-37.80^{\circ}$ & $-84.43^{\circ}$ \\
\hline
\end{tabular}

TABLE III

Solution of $\mathbf{G}_{1} \mathbf{W}_{1}$ With A SQUARE INITIAL CONFIGURATION

\begin{tabular}{|c||c||c|}
\hline Pivots & $\mathbf{G}_{1}$ & $\mathbf{W}_{1}$ \\
\hline 1 & $(0,0)$ & $(0,-1)$ \\
\hline $\mathbf{2}$ & $(\mathbf{0 . 5 5 7}, \mathbf{0 . 1 3 4})$ & $(\mathbf{0 . 8 8 8},-\mathbf{0 . 2 2 3})$ \\
\hline 3 & $(0.61-0.68 i, 0.51+0.62 i)$ & $(1.17-0.64 i, 0.37+0.17 i)$ \\
\hline 4 & $(0.61+0.68 i, 0.51-0.62 i)$ & $(1.17+0.64 i, 0.37-0.17 i)$ \\
\hline
\end{tabular}

and apply symmetry conditions such that the joint angles at each of the task position, $\theta_{2, j}=\theta_{3, j}=\cdots=\theta_{5, j}=\lambda_{j}, j=$ $1, \ldots, 5$. Also, if we let $\theta_{1, j}=\sigma_{j}, j=1, \ldots, 5$, we are able to solve for $\sigma_{j}, \lambda_{j}$, and $\theta_{6, j}$ at each of the task positions by equating the forward kinematics equation (25) with the task $\left[T_{j}\right], j=1 \ldots, 5$. See Table II.

Once we solve the inverse kinematics, the positions of its links $B_{1, j}, B_{2, j}, \ldots, B_{6, j}, j=1, \ldots, 5$ in each of the task positions can be determined. This means that we can identify five positions $\left[T_{j}^{B_{2}}\right], j=1, \ldots, 5$, when the end-effector is in each of the specified task positions. These five positions become our task positions in computing the RR chain $\mathbf{G}_{1} \mathbf{W}_{1}$ using (22) to constrain $B_{2}$ to ground. Note that since $B_{0}$ is the ground, $\left[D_{1 j}\right]$ reduces to $\left[B_{2, j}\right]\left[B_{2,1}\right]^{-1}, j=1, \ldots, 5$.

Similarly, we compute the RR chain $\mathbf{G}_{2} \mathbf{W}_{2}$ using (22) with $\left[D_{1 j}\right]=\left[B_{4,1}\right]\left[B_{4, j}\right]^{-1}\left[B_{6, j}\right]\left[B_{6,1}\right]^{-1}$.

We used this synthesis methodology and the five task positions listed in Table I to compute the pivots of the RR

TABLE IV

SOLUTION OF $\mathbf{G}_{2} \mathbf{W}_{2}$ WITH A SQUARE INITIAL CONFIGURATION

\begin{tabular}{|c||c||c|}
\hline Pivots & $\mathbf{G}_{2}$ & $\mathbf{W}_{2}$ \\
\hline 1 & $(0,0)$ & $(0,-1)$ \\
\hline 2 & $(0.239,-0.639)$ & $(0.097,-1.063)$ \\
\hline $\mathbf{3}$ & $(\mathbf{1 . 7 7 2},-\mathbf{0 . 2 9 8})$ & $(\mathbf{0 . 5 0 6},-\mathbf{1 . 2 7 2})$ \\
\hline 4 & $(3.962,17.757)$ & $(0.243,-0.812)$ \\
\hline
\end{tabular}

TABLE V

FIVE TASK POSITIONS FOR THE END-EFFECTOR OF THE PLANAR 6R ROBOT WITH A HEXAGON INITIAL CONFIGURATION

\begin{tabular}{|c||c|}
\hline Task & Position $(\phi, x, y)$ \\
\hline 1 & $\left(0^{\circ},-1,0\right)$ \\
\hline 2 & $\left(-39^{\circ},-0.15,2\right)$ \\
\hline 3 & $\left(-61^{\circ}, 0.8,2.35\right)$ \\
\hline 4 & $\left(-94^{\circ}, 1.75,2.3\right)$ \\
\hline 5 & $\left(-117^{\circ}, 2.25,2.1\right)$ \\
\hline
\end{tabular}

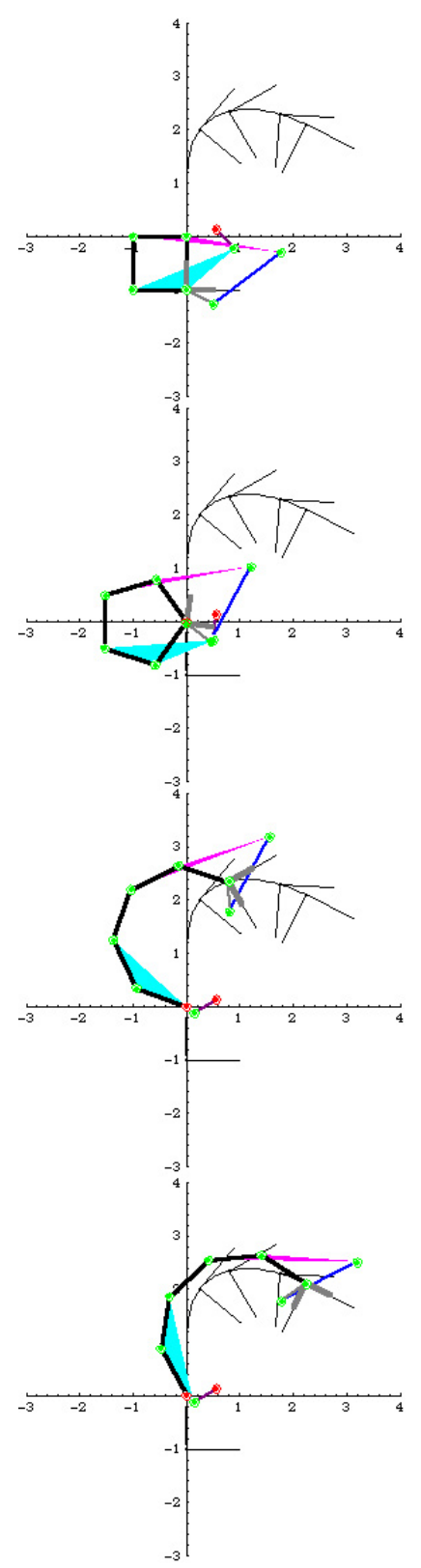

Fig. 10. This mechanically constrained $6 \mathrm{R}$ chain guides the end effector through five specified task positions with a square initial configuration

chains. We obtained two real solutions for $\mathbf{G}_{1} \mathbf{W}_{1}$, and four real solutions for $\mathbf{G}_{2} \mathbf{W}_{2}$, which result in one of the existing link for each case. Table III, and IV show the solution of the various possible pivots. The required transmission design and tendon routing is shown in Figure 5 and 6 respectively.

We follow the analysis procedure described in IX to animate the movement of the constrained 6R robot. See Figure 10.

\section{B. Example with a hexagon initial configuration}

We follow the procedure described in the earlier section to constrain the $6 \mathrm{R}$ chain with a hexagon initial configuration to one degree-of-freedom. Table $\mathrm{V}$ represents the movement of the manipulator of the $6 \mathrm{R}$ planar robot from a hexagon folded 
TABLE VI

Solution of $\mathbf{G}_{1} \mathbf{W}_{1}$ With a HEXAGON INITIAL CONFIGURATION

\begin{tabular}{|c||c||c|}
\hline Pivots & $\mathbf{G}_{1}$ & $\mathbf{W}_{1}$ \\
\hline 1 & $(0,0)$ & $(0.5,-0.866)$ \\
\hline 2 & $(-0.019,0.030)$ & $(3.966,-24.384)$ \\
\hline 3 & $(0.015,-0.006)$ & $(0.396,-0.442)$ \\
\hline $\mathbf{4}$ & $(\mathbf{0 . 1 7 9},-\mathbf{0 . 0 7 0})$ & $(-\mathbf{0 . 1 7 0}, \mathbf{0 . 1 4 1})$ \\
\hline
\end{tabular}

TABLE VII

Solution of $\mathbf{G}_{2} \mathbf{W}_{2}$ With a HeXAGON Initial CONFIGURATION

\begin{tabular}{|c||c||c|}
\hline Pivots & $\mathbf{G}_{2}$ & $\mathbf{W}_{2}$ \\
\hline 1 & $(-1.5,-0.866)$ & $(-1,0)$ \\
\hline $\mathbf{2}$ & $(-\mathbf{1 . 3 7 9 , 0 . 2 5 0})$ & $(-\mathbf{1 . 2 1 8 , 0 . 1 3 1})$ \\
\hline 3 & $(-1.22-0.20 i, 0.05-0.39 i)$ & $(-1.04-0.04 i,-0.01+0.05 i)$ \\
\hline 4 & $(-1.22+0.20 i, 0.05+0.39 i)$ & $(-1.04+0.04 i,-0.01-0.05 i)$ \\
\hline
\end{tabular}

state to its extended state. We solve the inverse kinematics of the $6 \mathrm{R}$ chain at each of the task positions by equating the forward kinematics equation (25). Once we solve the inverse kinematics, the positions of its links was used to design RR chain $\mathbf{G}_{1} \mathbf{W}_{1}$, and $\mathbf{G}_{2} \mathbf{W}_{2}$. We obtained four real solution for $\mathbf{G}_{1} \mathbf{W}_{1}$, and two real solutions for $\mathbf{G}_{2} \mathbf{W}_{2}$. Table VI, and VII show the various possible pivots. Again, we use the same transmission design and tendon routing as shown in Figure 5 and 6 respectively.

We follow the analysis procedure described in IX to animate the movement of the constrained 6R robot. See Figure 11

\section{CONCLUSIONS}

In this paper, we show that the design of a cable drive train integrated with four-bar linkage synthesis yields new opportunities for the constrained movement of planar serial chains. In particular, we obtain a $6 \mathrm{R}$ planar chain through five arbitrary task positions with one degree-of-freedom. The same theory applies to $\mathrm{nR}$ chains with more degrees of freedom. Two examples show that the end-effector moves through the specified positions, while the system deploys from square and hexagonal initial configurations.

\section{REFERENCES}

[1] J.J. Lee, Tendon-Driven Manipulators: Analysis, Synthesis, and Control, $\mathrm{PhD}$. dissertation, Department of Mechanical Engineering, University of Maryland, College Park. MD; 1991.

[2] M. Arsenault and C. M. Gosselin, "Kinematic and Static Analysis of a Planar Modular 2-DoF Tensegrity Mechanism", Proceedings of the Robotics and Automation, Orlando, Florida, 2006, pp. 4193-4198.

[3] M. J. Lelieveld and T. Maeno, "Design and Development of a 4 DOF Portable Haptic Interface with Multi-Point Passive Force Feedback for the Index Finger", Proceedings of the Robotics and Automation, Orlando, Florida, 2006, pp. 3134-3139.

[4] G. Palli and C. Melchiorri, "Model and Control of Tendon-Sheath Transmission Systems", Proceedings of the Robotics and Automation, Orlando, Florida, 2006, pp. 988-993.

[5] Krovi, V., Ananthasuresh, G. K., and Kumar, V., "Kinematic and Kinetostatic Synthesis of Planar Coupled Serial Chain Mechanisms," ASME Journal of Mechanical Design, 124(2):301-312, 2002.

[6] A. S. K. Kwan and S. Pellegrino, "Matrix Formulation of MacroElements for Deployable Structures," Computers and Structures, 15(2):237-251, 1994.

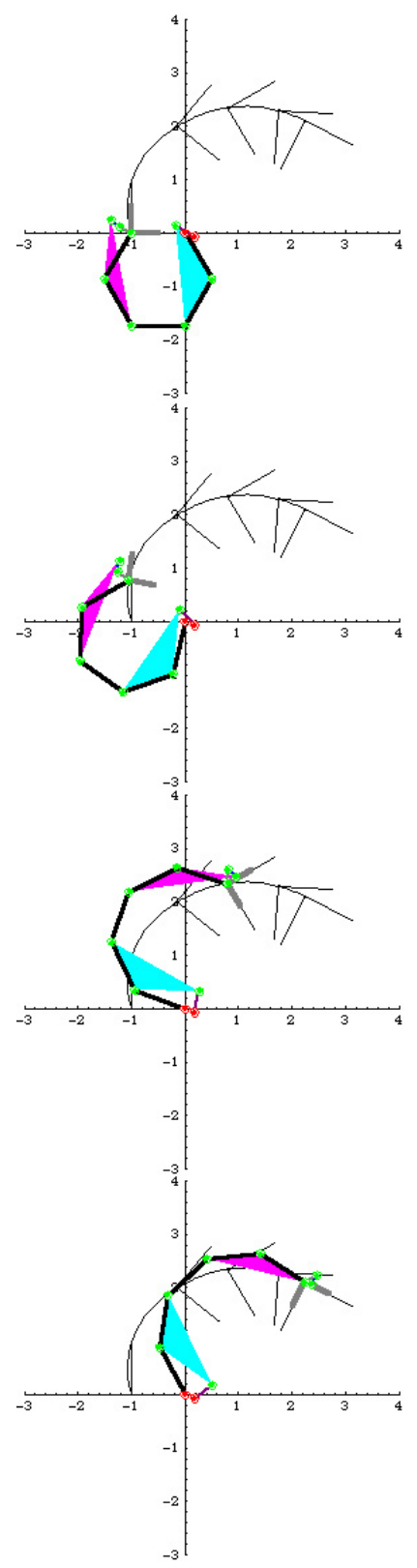

Fig. 11. This mechanically constrained $6 \mathrm{R}$ chain guides the end effector through five specified task positions with a hexagon initial configuration

[7] S. O. Leaver and J. M. McCarthy, "The Design of Three Jointed Two-Degree-of-Freedom Robot Fingers," Proceedings of the Design Automation Conference, vol. 10-2, pp. 127-134, 1987.

[8] S. O. Leaver, J. M. McCarthy and J. E. Bobrow, "The Design and Control of a Robot Finger for Tactile Sensing," Journal of Robotic Systems, 5(6):567-581, 1988.

[9] L.W. Tsai, Robot Analysis: The Mechanics of Serial and Parallel Manipulators, John Wiley \& Sons Inc, NY; 1999.

[10] G.N. Sandor and A.G. Erdman, A., Advanced Mechanism Design: Analysis and Synthesis, Vol. 2. Prentice-Hall, Englewood Cliffs, NJ; 1984.

[11] J. M. McCarthy, Geometric Design of Linkages, Springer-Verlag, New York; 2000. 